\title{
Stem Cells Nurturing Our Today and Tommorrow
}

\author{
${ }^{1}$ Dr. Poonam Malik, ${ }^{2}$ Dr. Ravi Sehravat, ${ }^{3}$ Dr. Jyotirmay Singh, \\ ${ }^{4}$ Dr. Manu Rathee \\ ${ }^{I}$ B.D.S.,M.D.S. Department Of Prosthodontics Sgt Dental College And Research Institute, Gurgaon Haryana \\ ${ }^{2}$ M.B.B.S, ,M.D.(Pharmacology) \\ ${ }^{3}$ B.D.S. ,M.D.S. Senior Lecturer Department Of Orthodontics And Dentofacial Orthopedics,Dr. B.R. Ambedkar \\ Institute Of Dental Sciences \& Research, Patna. \\ ${ }^{4}$ M.D.S., D.N.B., MNAMS, FPFA, FICD Senior Professor And Head Of Department, Department Of \\ Prosthodontics And Crown \& Bridge Govt. Dental College Pt.B.D.Sharma Post Graduate Institute Of Medical \\ Sciences (PGIMS)Pt. B.D.Sharma University Of Health Sciences Rohtak, Haryana
}

\begin{abstract}
Stem cell biology is currently one of the most exciting areas of biomedical research, as enthusiasm for the application of this technology toward regenerative medicine continues to expand. The application of stem cells in a therapeutic fashion may become a natural extension of the presumed potential of these unique cell populations with wide-ranging capabilities. As with many new and exciting technologies, much remains to be tested, proved, and delivered to separate the hope from the hype. In this review, we attempt to deliver the current "state of the art" in stem cell research and to provide a conceptual framework that can be used by surgeons as a basis for critical assessment of this quickly expanding and fascinating field.
\end{abstract}

\section{Introduction}

Stem cells have long been regarded as undifferentiated cells capable of proliferation, self-renewal, production a large number of differentiated progeny, and regeneration of tissues.1 Stem cells first became a topic of widespread public attention, when President George W. Bush authorized the use of federal funds for research on existing embryonic stem cell lines, but prohibited the derivation of new ones. Since that time, struggles over the types of stem cell research that should be allowed, and the levels of funding committed to such research, have continued unabated.

Stem cell research has been hailed for the potential to revolutionize the future of medicine and dentistry with the ability to regenerate damaged and diseased organs and tissues.. This article presents an overview of what stem cells are, what roles they play in normal processes such as development, repair, regeneration and how stem cells could have the potential to treat incurable diseases. Ethical issues are not the subject of this review. ${ }^{2-6}$

\section{Stem cells defined}

Stem cells are unspecialized cells that develop into the specialized cells that make up the different types of tissue in the human body. They are vital to the development, growth, maintenance, and repair of our brains, bones, muscles, nerves, blood, skin, and other organs. In the laboratory, researchers are learning how to coax stem cells to differentiate into specialized kinds of cells, and to create the conditions under which stem cells will replicate themselves for extended periods of time. If these unique properties can be understood and harnessed, stem cells hold great potential as tools for medical research and as therapeutic agents.

There are two main types of stem cells:?

- Embryonic stem cells are found in embryos at a very early stage of development. They have the ability to differentiate into any of the over two hundred types of cells that make up the human body.

- Adult stem cells have the ability to differentiate into varieties of a particular type of cell, determined by the type of tissue in which they are found. For example, blood stem cells found in the bone marrow give rise to red blood cells, white blood cells, and platelets.

Difference between the adult stem cells and embroyonic stem cells

The distinction is made between embryonic and adult stem cells.

Embryonic stem cells are pluripotent, that is, they can differentiate into all types of somatic cells and theoretically divide an unlimited number of times. ${ }^{8}$ Using immunosurgery, they can be harvested from early blastocyst stages (i. e., at about the 4th day of embryonic development). During this procedure, the blastocyst's trophoblasts are destroyed by an antibody-activated complement reaction. The embryoblast cells - the part of the blastocyst that is of interest for stem cell research - are maintained and, thanks to their ability to selfregenerate (which is typical of stem cells), can be multiplied. Their pluripotency remains intact. Through 
variations in growth factors, they can be specifically differentiated ${ }^{9,10,11}$ Ethically, the use of human embryonic cells is a highly contentious matter, because harvesting them requires the destruction of human embryos early in development. Up to now, only isolated studies on animal embryonic stem cells have been conducted ${ }^{12,13,14,15}$

Tissue samples from various "parent" tissues can serve as the source for harvesting adult stem cells. Adult stem cells can only proliferate a limited number of times. They are distinguished according to their developmental potential. There are uni- and bipotent progenitor cells, which can usually only be differentiated into mature cells of their parent tissue, and multipotent adult stem cells, which can also differentiate into tissues that are not identical to the parent tissue. ${ }^{8}$ Adult stem cells are theoretically present in every type of tissue. Organs that are particularly suited for yielding adult stem cells include the bone marrow (Pittenger et al. 1999), ${ }^{16}$ the umbilical cord, and umbilical cord blood (Noll 2003). ${ }^{17}$ In order to minimize the lesion inflicted by taking the tissue sample and limit the weakening of the organ or organism, the concentration of stem cells in the obtained tissue sample should be as large as possible..$^{18,19}$

\section{Stem cell and medicine applications}

Stem cell technologies have, or are anticipated to have, applications for basic science, medical research, and therapies. ${ }^{20,21}$

- $\quad$ Basic science applications. Stem cells are ideally suited to allow for the study of complex processes that direct early unspecialized cells to differentiate and develop into the more than two hundred cell types in the human body.

- Medical research applications. Stem cell studies may allow researchers to follow the processes by which diseases and impairments caused by genetic abnormalities first manifest themselves biochemically or structurally in cells and tissues. Using stem cells to produce large numbers of genetically uniform cultures of organ tissues - for example, liver, muscle, or neural-would allow controlled comparison of the effects of drugs or chemicals on these tissues.

Alternatively, testing drugs against stem cell tissues of varying genetic makeup could allow development of pharmaceuticals tailored to provide greater benefits, and with fewer side effects, for patients with specific gene-related characteristics. In addition, the use of human stem cell cultures might reduce the need to use animals for research and testing purposes.

- Therapeutic applications. The prospect of using stem cells to repair or replace damaged or diseased tissues has generated enormous interest. In the courses of their lives, the great majority of people suffer from one degenerative condition or another.

The conditions that stem cell technologies might conceivably address include Parkinson's disease, spinal cord injury, stroke, type 1 diabetes, heart disease, rheumatoid arthritis, osteoarthritis, kidney disease, blood diseases (including sickle cell anemia), blindness, muscular dystrophy, liver disease, loss of teeth, and baldness. Some researchers have speculated that stem cell technologies might allow entire organsstomachs, hearts, livers, kidneys, and others - to be grown and used for transplantation. Stem cells also might be used in conjunction with other therapies. For example, they might be used to replenish immune cells destroyed during chemotherapy for cancer.

\section{Therapeutic Repair Strategies: The "R3" Paradigm}

The scope of stem cell-based regenerative medicine is defined by the convergent repair triad of replacement, regeneration, and rejuvenation. The "R3" paradigm of therapeutic repair highlights that these strategies overlap in practice while inherent distinctions conceptualize the scope of regenerative medicine, ranging from transplantation of used parts ("replacement") to development of new parts ("regeneration") to induction of self-renewed parts ("rejuvenation").

\section{Replacement}

Replacement strategy refers to transplantation of a cell-based product that re-establishes homeostasis for the recipient through continuation of the tissue function from the donor. The field of surgery pioneered the concept of replacement with the advent of solid organ transplantation. If the heart was damaged beyond the ability to palliate the condition, then replacing the diseased tissue with a functioning donor heart became the only option. In addition to solid organ transplantation, cell-based replacement is routinely used in the form of red blood cell transfusions to replace the circulating blood in order to increase the oxygen-carrying capacity and treat life-threatening blood loss or anemia. This strategy "recycles" used parts of cells, tissues, or organs to "restore" physiologic function. A significant limitation of the replacement strategy remains the shortage of appropriate donors and the difficulty to match the immunological criteria for a safe and effective transplantation. 


\section{Regeneration}

Regenerative strategy refers to engraftment of progenitor cells that require in vivo growth and differentiation to establish recipient homeostasis through de novo function of the stem cellbased transplant. Advances in hematology gave rise to the concept of regeneration with the identification of bone marrow-derived stem cells that once harvested could be transplanted in small quantities into the peripheral blood to engraft and reconstitute the functioning bone marrow through continuous production of the entire hematopoietic system. Success was facilitated by the presence of host bone marrow that provided a protective environment to nurture the long-term survival of self-renewing stem cell progenitors. This strategy "restores" function by "renewing" the pool of functional progenitor cells to allow differentiation as needed from exogenous stem cells. An intense search is ongoing for tissue-specific, nonhematopoietic stem cells that have the capacity to re-establish lost function when ectopically transplanted into a wide range of diseased tissues, as evident in diabetes, ischemic heart disease, and degenerative neurological diseases. ${ }^{23}$

\section{Rejuvenation}

Rejuvenation strategy refers to self-renewal of tissues from endogenous, resident stem cells to maintain tissue homeostasis and promote tissue healing. This natural process of tissue recycling enables cells as they senesce to be replaced with younger cells that are inherently more resilient and equipped to provide adequate stress tolerance for tissue survival. Daughter cells can also be derived from reactivation of the cell cycle within mature cell types in response to (physio) pathological stress. This strategy "renews" tissue structure by "recycling" endogenous stem cells for proactive self-renewal. Rejuvenation ensures continuous production of renewable tissue required for long-term stress tolerance; however, most tissues are able to only partially selfrenew. In the context of a massive acute injury, such as myocardial infarction, an inherent repair strategy may be inadequate.19 A boost in these natural processes, through biologic or pharmacologic treatment, is likely required to stimulate adaptive response and promote adequate biogenesis of functional tissue in the setting of acute or progressive disease. ${ }^{24}$

\section{Stem cells and dentistry-a NEW HOPE}

Dental exfoliation in humans is a genetically regulated event during childhood. If the permanent teeth are damaged or lost, they do not regenerate. At present, teeth can only be replaced with conventional prostheses, i. e., removable prostheses, fixed dental prostheses, or implants, with prior bone augmentation if necessary. However, progress in stem cell biology and tissue engineering may present new options for replacing heavily damaged or lost teeth, or even individual tooth structures. The promise of such treatment possibilities puts stem cells in the focus of dental research.

The ultimate goal of tooth regeneration is to replace the lost teeth. Stem cell-based tooth engineering is deemed as a promising approach to the making of a biological tooth (bio-tooth). Dental pulp stem cells represent a kind of adult cell colony which has the potent capacity of self-renewing and multilineage differentiation. The exact origin of Dental pulp stem cells has not been fully determined and these stem cells seem to be the source of odontoblasts that contribute to the formation of dentin-pulp complex. ${ }^{25}$

Recently, achievements obtained from stem cell biology and tooth regeneration have enabled us to contemplate the potential applications of Dental pulp stem cells. Some studies have proved that Dental pulp stem cells are capable of producing dental tissues in vivo including dentin, pulp, and crown-like structures. Whereas other investigations have shown that these stem cells can bring about the formation of bone-like tissues. Theoretically, a bio-tooth made from autogenous Dental pulp stem cells should be the best choice for clinical tooth reconstruction.

Some initial success with dental tissue indicates that stem cell research may be of therapeutic use in dentistry as well, for instance, to regenerate individual tissue types, such as bone ${ }^{26,27,28,29}$, periodontal tissue $30,31,32$, or someday even entire teeth ${ }^{33,34}$.

Fundamentally, two means of regenerating teeth are described.

$>$ The first is conventional tissue engineering, in which the application of cells in a carrier material in vitro under the influence of a stimulus leads to tissue regeneration.

$>$ The second is the much more innovative process of tooth regeneration using dental epithelium and mesenchymal cells in vivo after direct implantation, representing a kind of tissue engineering in the broader sense (Wang \& Wang 2008), based on knowledge of general embryogenesis and physiological tooth development during childhood.35

No systematic literature review exists yet on the topic of "implementation of stem cell biology in tooth development". 


\section{In dentistry - Current and future developments}

Although the future of dentistry is highly speculative, dental stem cells will revolutionize dentistry in the near future. In a reasonable period, we cannot exclude, that dental stem cells for example will support paradontology or procedures for bone augmentation. Stem cell scientists are very often asked for novel bioengineered dentures. linical applications of dental stem cells will continue to emerge in the near term and longer term. Currently dental stem cell research focuses on regeneration of dentine, pulp and teeth; alveolar bone; regeneration of periodontal ligament after periodontal disease; salivary gland regeneration after radiation therapy; repair of craniofacial defects; and in the treatment of lichen planus. ${ }^{32}$ Although there is no known example in patients, patents describe methods to create bio-dentures. However, the realization seems to be problematic also due to parameters that are not easy to control such as the high risk of rejection and long tooth eruption period. These problems cannot be solved in a short time, but technical progress and dental stem cell research may workout solutions in the future.

\section{Advantages of stem cells}

Stem cells can be engineered to replicate various specialized cells---those in the brain, liver and skin--and have the potential to treat vast numbers of illnesses. There is a strong likelihood that stem cells can generate healthy organs in a laboratory, to be transplanted into people needing them.

Stem cell research is especially exciting because it gives new hope to people who have terminal or incurable conditions. For instance, while Parkinson's patients take drugs that slow or lessen their symptoms, the drugs are incapable of curing the diseased tissue in the brain. This is where stem cell research comes in, because it has the capability to generate disease-free tissue. Parkinson's is just one of many diseases poised to benefit from stem cell treatment.

\section{Demerits of stem cells}

The opponents of human embryonic stem cell research reacted by pointing out that the considerable ethical problem is the source of those stem cells. That living human embryos as the most vulnerable human beings are set to be destroyed while taking those stem cells out of their bodies for research. That it is never acceptable in the field of ethics to kill any innocent human being intentionally even if it promises benefit to the majority. It is said to be tantamount to reducing these fetuses and embryos to mere objects for others' use, instead of subjects with inherent rights that must be given equal protection.

However, in spite of the above merits and demerits, scientists are continuously working on stem cell research, in order to save the lives of millions of people. ${ }^{36}$

\section{Summary}

As the political debate about stem cell research continues, the scientific discoveries and substantiation of earlier claims will proceed. Obvious potential clinical benefits may result from much of this work, but in a larger sense the rethinking of long-held biological paradigms may prove to be ultimately as valuable. The concerns voiced by others to proceed with caution and await the rigors demanded by good science should be the precedent. The alternative is to allow the hype to embolden claims and hopes that may not be deliverable if a "stem cell bubble" goes unchecked. A great deal of basic research is needed to further explore the current candidate cell populations before potential clinical benefits of stem cell research can begin to be realized.

\section{References}

[1]. Asahara T, Kalka C, Isner JM. Stem cell therapy and gene transfer for regeneration. Gene Ther. 2000;7(6):451-457

[2]. Bianco P, Riminucci M, Gronthos S, Robey PG Bone marrow stromal stem cells: nature, biology, and potential applications. Stem Cells 2001;19:180-192.

[3]. Greco SJ, Liu K, Rameshwar P .Functional similarities among genes regulated by Oct4 in human mesenchymal and embryonic stem cells. Stem Cells .2007; 25:3143-3154.

[4]. Jones DL, Wagers AJ. No place like home: anatomy and function of the stem cell niche. Nat Rev Mol Cell Biol .2008; 9:11-21

[5]. Jorgensen C, Djouad F, Apparailly F, Noel D . Engineering mesenchymal stem cells for immunotherapy. Gene Ther .2003;10:928931

[6]. Baksh D, Song L, Tuan RS.Adult mesenchymal stem cells: characterization, differentiation, and application in cell and gene therapy. J Cell Mol Med .2004;8:301-316

[7]. Mao JJ. Stem Cells and the Future of Dental Care. N Y State Dent J. 2008;74:20-4.

[8]. Morsczeck C, Reichert T E, Vollner F, Gerlach T, Driemel O: The state of the art in human dental stem cell research. Mund Kiefer Gesichtschir.2007;11: 259-266

[9]. Cowan C A, Klimanskaya I, McMahon J, Atienza J, Witmyer J, Zucker J P, Wang S, Morton C C, McMahon A P, Powers D, Melton D A: Derivation of embryonic stem-cell lines from human blastocysts. N Engl J Med .2004;350: 1353-1356.

[10]. Terskikh A V, Bryant P J, Schwartz P H: Mammalian stem cells. Pediatr Res .2006;59: 13R-20R.

[11]. Ding G, Wang W, Liu Y, Zhang C, Wang S. Mesenchymal stem cell transplantation: A potential therapy for oral lichen planus. Med Hypotheses. 2011;76:322-4.

[12]. Honda M J, Fong H, Iwatsuki S, Sumita Y, Sarikaya M: Tooth-forming potential in embryonic and postnatal tooth bud cells. Med Mol Morphol.41: 183-192 (2008) 
[13]. Kang H K, Roh S, Lee G, Hong S D, Kang H, Min B M: Osteogenic potential of embryonic stem cells in tooth sockets. Int J Mol Med .2008;21: 539-544.

[14]. Zhu F, Nie R R, Wu L, Liu L, Tang W, Tian W D: Spontaneous odontogenic differentiation and critical gene expression of mouse dental papilla mesenchymal cell in vitro. Sichuan Da Xue Xue Bao Yi Xue Ban .2008;39: 286-289, 297.

[15]. Ikeda E, Morita R, Nakao K, Ishida K, Nakamura T, Takano-Yamamoto T, Ogawa M, Minuzo M, Kasugai S, Tsuji T: Fully functional bioengineered tooth replacement as an organ replacement therapy. PNAS.2009; 106: 13475-13480.

[16]. Nosrat IV, Widenfalk J, Olson L, Nosrat CA. Dental pulp cells produce neurotrophic factors, interact with trigeminal neurons in vitro, and rescue motoneurons after spinal cord injury. Dev Biol. 2001;238:120-32.

[17]. Nagaya N, Fujii T, Iwase T, Ohgushi H, Itoh T, Uematsu M, et al. Intravenous administration of mesenchymal stem cells improves cardiac function in rats with acute myocardial infarction through angiogenesis and myogenesis. Am $\mathrm{J}$ Physiol Heart Circ Physiol. 2004;287:H2670-6.

[18]. Thomson JA, Itskovitz-Eldor J, Shapiro SS, Waknitz MA, Swiergiel JJ, Marshall VS, et al. Embryonic stem cell lines derived from human blastocysts. Science. 1998;282:1145-7.

[19]. Asahara T, Kalka C, Isner JM. Stem cell therapy and gene transfer for regeneration. Gene Ther. 2000;7(6):451-457

[20]. Atala A. Advances in tissue and organ replacement. Curr Stem Cell Res Ther 2008;3:21-31.

[21]. Kørbling M, Estrov Z. Adult stem cells for tissue repair-a new therapeutic concept? N Engl J Med 2003;349:570-582.

[22]. Surani MA, McLaren A. Stem cells: a new route to rejuvenation. Nature 2006;443:284-285.

[23]. Bluteau G, Luder H U, De Bari C, Mitsiadis T A: Stem cells for tooth engineering. Eur CellMater.2008; 16: 1-9.

[24]. de Mendonca Costa A, Bueno D F, Martins M T, Kerkis I, Kerkis A, Fanganiello R D, Cerruti H, Alonso N, Passos-Bueno M R: Reconstruction of large cranial defects in nonimmunosuppressed experimental design with human dental pulp stem cells. $\mathrm{J}$ Craniofac Surg .2008;19: 204-210.

[25]. Graziano A, d'Aquino R, Laino G, Proto A, Giuliano M T, Pirozzi G, De Rosa A, Di Napoli D, Papaccio G: Human CD34+ stem cells produce bone nodules in vivo. Cell Prolif .2008;41: 1-11 (2008)

[26]. Pittenger MF, Mackay AM, Beck SC, Jaiswal RK, Douglas R, Mosca JD, et al. Multilineage potential of adult human mesenchymal stem cells. Science. 1999;284:143-7.

[27]. Zheng Y, Liu Y, Zhang C M, Zhang H Y, Li W H, Shi S, Le A D, Wang S L: Stem cells from deciduous tooth repair mandibular defect in swine. J Dent Res.2009; 88: 249-254

[28]. Shi S, Bartold P M, Miura M, Seo B M, Robey P G, Gronthos S: The efficacy of mesenchymal stem cells to regenerate and repair dental structures. Orthod Craniofac Res .2005;8: 191-199.

[29]. Silverio K G, Benatti B B, Casati M Z, Sallum E A, Nociti F H Jr: Stem cells: potential therapeutics for periodontal regeneration. Stem Cell Rev.2008; 4:13-19.

[30]. P.S. Shilpa, R Kaul,N Sultana,S Bhat. Stem cells: Boon to dentistry and medicine .Dent Res J (Isfahan). 2013 Mar-Apr; 10(2): 149154.

[31]. Xu L, Tang L, Jin F, Liu X H, Yu J H, Wu J J, Yang Z H, Wang Y X, Duan Y Z, Jin Y: The apical region of developing tooth root constitutes a complex and maintains the ability to generate root and periodontium-like tissues. J Periodontal Res.2009; 44: $275-282$.

[32]. Singh J. Stem cells nurturing our future. Patna J medicine.2011;85(2):17-21.

[33]. Jung SH, Ryu JI, Jung DB. Association of total tooth loss with socio-behavioural health indicators in Korean elderly. J Oral Rehabil. 2010;38:517-24.

[34]. Wang S L, Wang X J: Tooth regeneration - dream to reality. Hua Xi Kou Qiang Yi Xue Za Zhi.2008; 26: 115-117.

[35]. Meyer JR. Human embryonic stem cells and respect for life. J Med Ethics. 2000;26(3):166-170.

[36]. Ferrari G, Cusella-De Angelis G, Coletta M, Paolucci E, Stornaiuolo A, Cossu G, et al. Muscle regeneration by bone marrowderived myogenic progenitors. Science.1998;279:1528-30. 